Pacific Journal of Mathematics

INVARIANT SUBSPACES, SIMILARITY AND ISOMETRIC
EQUIVALENCE OF CERTAIN COMMUTING OPERATORS IN

ROBERT E. WATERMAN 


\section{INVARIANT SUBSPACES, SIMILARITY AND ISOMETRIC EQUIVALENCE OF CERTAIN COMMUTING OPERATORS IN $L_{p}$}

\section{ROBERT E. WATERMAN}

This paper is concerned with the problem of finding all closed invariant subspaces of operators of the form $T_{f}=$ $M_{f}-J M_{f}$, and the determination of the similarity relationships between such operators. The operator $T_{f}$ is defined, for suitable conditions on a complex-valued function $f$ and its derivative $f^{\prime}$, by $T_{f} g(x)=f(x) g(x)-\int_{0}^{x} f^{\prime}(t) g(t) d t$ for all $g$ in $L_{p}$. The main result asserts that the closed invariant subspaces of $T_{f}$ are precisely those subspaces that are generated by the eigenfunctions of $T_{f}$. Conversely, any operator on $L_{p}$ whose closed invariant subspaces coincide with those of $M-J$ (i.e., $T_{f}$ where $f(x) \equiv x$ ) must be of the form $T_{g}$ for some function $g$. The closed invariant subspaces of $T_{f}$ are cyclic and the generating functions have a rather simple description. The algebra of operators $\mathscr{C}$, generated by $M-$ $J$, is maximal abelian. A corollary is that $\mathscr{C}$ is reflexive. It is shown that $T_{f}$ and $T_{g}$ are isometrically equivalent in $L_{p}$ if and only if $f=g$. Finally conditions are given for the similarity of $T_{f}$ and $T_{g}$.

We give a brief history of the problem of finding all closed invariant subspaces of a given class of operators. The case of cyclic hermitian operators is well-known; an exposition appears in Plesner [8]. The general case is accessible via multiplicity theory (see Plesner, loc. cit.). Beurling [1], in an important paper, found all the closed invariant subspaces of the shift operator on $\ell_{2}$ through a detailed study of certain analytic functions on the unit disc. Donoghue [3] found all closed invariant subspaces of the simple Volterra operator $J$, defined by $f \rightarrow \int_{0}^{x} f$, on $L_{2}(0,1)$ and those of a certain weighted shift operator. Kalisch [7] found all closed invariant subspaces of an extensive class of integral operators (including $J$ ) on $L_{p}(0,1)$ for $1<p<\infty$. Here we examine the invariant subspace structure of $M-J$ (and more generally, $T_{f}=M_{f}-J M_{f^{\prime}}$ ). In spite of appearing superficially the same, the problem of finding all the closed invariant subspaces of $M-n J$ ( $n$ an integer $>1$ ) is quite different and considerably more difficult. This problem has been solved by the author (using recent results on $L_{p}$ approximation by splines) and will appear elsewhere.

1. Definitions. We are concerned with bounded operators on 
$L_{p}=L_{p}(0,1)$ for $1<p<\infty$. Define (for suitable functions $f$ and $r$ ) the multiplication operator $M_{f}$, the Volterra operator $J$, and the substitution operator $S_{r}$ respectively by $M_{f} g=f g, J g=\int_{0}^{x} g$ (with respect to Lebesgue measure) and $S_{r} g=g \circ r$ (composition of $g$ and $r$ ). For $f \in$ $L_{p}$ and $g \in L_{q}$, where $1 / p+1 / q=1$, we define the Banach inner product of $f$ and $g$ by $(f, g)=\int_{0}^{1} f g$. We write $B\left(L_{p}\right)$ for the algebra of bounded operators on $L_{p}$ and write $T^{*}$ to denote the Banach adjoint of $T \in B\left(L_{p}\right)$; i.e., $T^{*}$ is the unique member of $B\left(L_{q}\right)$ such that $(T f, g)=\left(f, T^{*} g\right)$ for all $f \in L_{p}$ and $g \in L_{q}$. In particular, we have $J^{*}=\int_{x}^{1}$ and $M_{f}^{*}=M_{f}$. If $W$ is a closed invariant subspace of $T \in B\left(L_{p}\right)$ then we will refer to the restriction of $T$ to $W$ as the part of $T$ on $W$ or simply as a part of $T$. For $a \in[0,1]$ we will write $e_{a}$ to denote the characteristic function $\chi_{[a, 1]}$ of the interval $[a, 1]$. If $E \cong[0,1]$ is a nonempty closed set we define $W(E)$ to be the closed subspace of $L_{p}$ generated by the set of functions $\left\{e_{a}: a \in E\right\}$. We will say that a complex-valued function $f:[0,1] \rightarrow C$ is absolutely continuous on $[a, b)$ if $f$ is absolutely continuous on every closed interval contained in $[a, b)$; the same convention will apply to the intervals $(a, b]$ and $(a, b)$.

If $f$ is a complex-valued function whose derivative $f^{\prime}$ exists a.e. then we will write $T_{f}$ to denote $M_{f}-J M_{f^{\prime}}$. We define $\mathscr{C}$ to be the collection of all operators $T_{f}$ such (i) $f \in L_{\infty}$ and $f$ is absolutely continuous on $[0,1)$, and (ii) the function $k_{f}(x) \equiv(1-x)^{q_{I} p} \int_{0}^{x}\left|f^{\prime}(t)\right|^{q} d t$ belongs to $L_{\infty}$. The fact that $\mathscr{C} \subseteq B\left(L_{p}\right)$ follows from a result in [2]. In fact, $T_{f} \in \mathscr{C}$ implies that $M_{f}$ and $J M_{f}$, each belong to $B\left(L_{p}\right)$. Condition (ii), above, implies that if $T_{f} \in \mathscr{C}$ then $f^{\prime}$ belongs to $L_{q}(0, a)$ (i.e., $\left.\int_{0}^{a}\left|f^{\prime}\right|^{q}<\infty\right)$ for all $a<1$; we will use this fact often in the sequel. We also note that (i) and (ii) together do not imply that $f$ is absolutely continuous on $[0,1]$. Indeed, it may happen that $T_{f} \in \mathscr{C}$ while $f$ fails to be continuous at $x=1$, as the example $f(x)=$ $\sin (-\log (1-x))$ shows. We define $\mathscr{A}$ to be the subset of $\mathscr{C}$ consisting of those operators $T_{f} \in \mathscr{C}$ such that $f$ is $1-1$ and absolutely continuous on $[0,1]$ and $m\left\{x \in[0,1]: f^{\prime}(x)=0\right\}=0$ (where $m$ denotes Lebesgue measure).

Our first lemma details the important algebraic properties of $\mathscr{C}$. Part (ii) establishes the fact that $\mathscr{C}$ is indeed an (abelian) algebra. The formula in part (iii) shows that $P\left(T_{r}\right)=T_{P(r)}$, for any polynomial $P$ and any member $T_{r}$ of $\mathscr{C}$. This operational calculus is easily extended to more general functions. Part (iv) of the lemma plays a crucial role in the proof of our main lemma (Lemma 4) on the invariant subspaces of $T_{f}$. The formula appearing in part (iv) becomes somewhat more transparent if one considers the special case $r(x) \equiv x$. 
Lemma 1. If $T_{r}$ and $T_{s}$ belong to $\mathscr{C}$ then:

(i ) $M_{r} J-J M_{r}=J M_{r} J$;

(ii) $T_{r}$ and $T_{s}$ commute and their product is equal to $T_{r s}$ which also belongs to $\mathscr{C}$;

(iii) $T_{r}^{n}=M_{r}^{n}-n J M_{r}, M_{r}^{n-1}$ for all $n \in Z^{+}$;

(iv) if $r(0)=0$ then $\left(T_{r}^{n} g, h\right)=n\left(r^{n-1},\left(M_{r^{\prime}} J^{*} M_{g}-M_{g} M_{r^{\prime}} J^{*}\right) h\right)$ for $n \in Z^{+}$and all $g \in L_{p}$ and $h \in L_{q}$.

Proof. (i) Let $g \in L_{p}$. Then since $r$ is absolutely continuous on $[0, x]$ for $:<1$ we may integrate by parts to compute

$$
\int_{0}^{x} r(t) g(t) d t+\int_{0}^{x} r^{\prime}(t) \int_{0}^{t} g(z) d z d t=r(x) \int_{0}^{x} g(t) d t,
$$

which, since $x<1$ and $g \in L_{p}$ were arbitrary, demonstrates that $J M_{r}+$ $J M_{r^{\prime}} J=M_{r} J$.

(ii) Using part (i) to evaluate $J M_{r^{\prime}} J$ we have

$$
\begin{aligned}
T_{r} T_{s} & =M_{r s}-M_{r} J M_{s^{\prime}}-J M_{r^{\prime}} M_{s}+\left(J M_{r^{\prime}} J\right) M_{s^{\prime}} \\
& =M_{r s}-J M_{r^{\prime}} M_{s}-J M_{r} M_{s^{\prime}} \\
& =M_{r s}-J M_{(r s)^{\prime}}=T_{r s} .
\end{aligned}
$$

Similarly we may show that $T_{s} T_{r}=T_{s r}$ and since $T_{r s}=T_{s r}$ we have proved that $T_{r}$ and $T_{s}$ commute with product equal to $T_{r s}$. The fact that $T_{r s} \in \mathscr{C}$ is a routine verification.

(iii) This follows immediately from part (ii).

(iv) Let $T=T_{r}$. Since $r(0)=0$ it is easily verified that $M_{r} e_{0}=$ $J M_{r}, e_{0}$ and thus for $n=1$ and any $g \in L_{p}$ and $h \in L_{q}$ we have

$$
\begin{aligned}
(T g, h) & =\left(M_{r} g, h\right)-\left(J M_{r^{\prime}} g, h\right) \\
& =\left(M_{g} J M_{r^{\prime}} e_{0}, h\right)-\left(J M_{r^{\prime}} M_{g} e_{0}, h\right) \\
& =\left(e_{0},\left(M_{r^{\prime}} J^{*} M_{g}-M_{g} M_{r^{\prime}} J^{*}\right) h\right) .
\end{aligned}
$$

We now sketch the inductive step, where the adjoint of (i) is used to evaluate the term $M_{r^{\prime}}\left(J^{*} M_{r^{\prime}} J^{*}\right)$ that arises in our computation. Let $N=J M_{r^{\prime}}$. Then

$$
\begin{aligned}
\left(T^{n+1} g, h\right) & =\left(T^{n} g, T^{*} h\right) \\
& =n\left(r^{n-1},\left(N^{*} M_{g} M_{r}-N^{*} M_{g} N^{*}-M_{g} N^{*} M_{r}\right) h\right) \\
& +n\left(r^{n-1}, M_{g} M_{r^{\prime}}\left(J^{*} M_{r^{\prime}} J^{*}\right) h\right) \\
& =n\left(r^{n-1},\left(N^{*} M_{g} M_{r}-N^{*} M_{g} N^{*}-M_{g} M_{r} N^{*}\right) h\right) \\
& =n\left(N r^{n-1}, M_{g}\left(M_{r}-N^{*}\right) h\right)-n\left(r^{n}, M_{g} N^{*} h\right) \\
& =(n+1)\left(N r^{n}, M_{g} h\right)-(n+1)\left(r^{n}, M_{g} N^{*} h\right) \\
& =(n+1)\left(r^{n},\left(M_{r^{\prime}} J^{*} M_{g}-M_{g} M_{r^{\prime}} J^{*}\right) h\right) .
\end{aligned}
$$


The next lemma provides us with a useful tool for deciding when a densely defined "formal" operator (i.e., one given by a formula, such as $M_{f}-J M_{f^{\prime}}$ ) that is capable of being extended to an "abstract" operator in $B\left(L_{p}\right)$ is actually defined everywhere in $L_{p}$ by the given formula. The rather straightforward proof, which may be based on a standard theorem in analysis [4, p. 156], is left to the reader.

LEMma 2. Let $T$ be a function from $L_{p}$ into the set of measurable functions on $[0,1]$. Suppose further that $T$ is bounded and linear on a dense subspace $D$ of $L_{p}$ and $T(D) \subseteq L_{p}$. Then $T$ maps $L_{p}$ into $L_{p}$ and is linear (and bounded) if and only if $f_{0} \in L_{p}$ and $\left\{f_{n}: n=1\right.$, $2, \cdots\} \subseteq D$ and $\left\|f_{n}-f_{0}\right\|_{p} \rightarrow 0$ together imply that there exists a subsequence $\left\{f_{n_{k}}\right\}$ such that $T f_{n_{k}} \rightarrow T f_{0}$ pointwise a.e. on $[0,1]$.

The following elementary fact is used repeatedly in the sequel and so we single it out as a lemma.

LEMMA 3. Let $E$ be a nonempty closed subset of $[0,1]$ and let $g \in L_{p}$. Then $\left(J^{*} g\right)(x)=0$ for all $x$ in $E$ implies that $g=0$ a.e. on $E$.

Proof. Let $h=-J^{*} g$ and define $F$ to be the set consisting of those points $x$ in $E$ such that $x$ is a limit point of $E$ and $h^{\prime}(x)=g(x)$. Clearly $m(F)=m(E)$ and so if $m(F)=0$ the lemma is trivial. If $m(F)>0$ and $x$ belongs to $F$ let $\left\{x_{n}\right\} \subseteq E$ be a sequence converging to $x$. Then $h(x)=h\left(x_{n}\right)=0$ so that

$$
g(x)=h^{\prime}(x)=\lim _{n \rightarrow \infty} \frac{h(x)-h\left(x_{n}\right)}{x-x_{n}}=0,
$$

which completes our proof.

2. Invariant subspaces. We now turn our attention to the classification of the closed invariant subspaces of operators that belong to the set $\mathscr{A}$. Our Main Lemma, which gives a characterization of the functions that belong to a given closed invariant subspace of an operator in $\mathscr{A}$, is used repeatedly in much of what follows.

LEMMA 4 (MAIN LEMMA). Let $W$ be a closed invariant subspace of $T_{f} \in \mathscr{A}$. Let $E=\left\{a \in[0,1]: e_{a} \in W\right\}$ and $K=\inf E$. Then

(i) $E$ is closed and nonempty;

(ii) if $f_{0} \in W$ then $f_{0}$ is a constant $k_{i}$ a.e. on each component $\left(c_{i}, d_{i}\right)$ of the open set $[K, 1] \backslash E=\bigcup_{i=1}^{\infty}\left(c_{i}, d_{i}\right)$ and $f_{0}=0$ a.e. on $[0, K]$.

Proof. (i) Since $1 \in E$ we have $E \neq \varnothing$. Letting $x_{i} \rightarrow x$ with $\left\{x_{i}\right\} \subseteq E$ one easily verifies that $\left\|e_{x_{i}}-e_{x}\right\|_{p} \rightarrow 0$. Thus $x \in E$ and so 
$E$ is closed.

(ii) Without loss of generality we may suppose that $f(0)=0$ since $T_{f}$ and $T_{(f-f(0))}=T_{f}-f(0)$ have the same invariant subspaces. If $K=0$ then trivially $f_{0}=0$ a.e. on $[0, K]$. Thus we will assume that $K>0$ for the remainder of the proof. Let $a \in U$ where $U=$ $(0, K)$ or $U=\left(c_{i}, d_{i}\right)$ is a component of $[K, 1] \backslash E$. Then $e_{a} \notin W$ and so by the Hahn-Banach Theorem there exists a continuous linear functional $S$ on $L_{p}$ such that $S(W)=0$ and $S\left(e_{a}\right) \neq 0$. According to the Riesz Representation Theorem there exists an $h$ in $L_{q}$ such that $S g=(g, h)$ for all $g$ in $L_{p}$. Thus $\left(T_{f}^{n} f_{0}, h\right)=0$ for all $n \in Z^{+}$and so Lemma 1 (iv) gives us

$$
0=\left(f^{n-1},\left(M_{f^{\prime}}, J^{*} M_{f_{0}}-M_{f_{0}} M_{f^{\prime}} J^{*}\right) h\right)
$$

for all $n \in Z^{+}$. Since $f$, by hypothesis, is continuous and 1-1, a theorem due to J. L. Walsh $[9$, p. 39] implies that polynomials in $f$ are dense in the space of complex-valued continuous functions on $[0,1]$ and consequently are dense in $L_{p}$. Thus (1) and $f^{\prime} \neq 0$ a.e. (since $\left.T_{f} \in \mathscr{A}\right)$ together imply that

$$
f_{0}(x) \int_{x}^{1} h(t) d t=\int_{x}^{1} f_{0}(t) h(t) d t
$$

almost everywhere. Now

$$
\int_{a}^{1} h(t) d t=\int_{0}^{1} e_{a}(t) h(t) d t=\left(e_{a}, h\right)=S e_{a} \neq 0
$$

and thus, since $\int_{x}^{1} h(t) d t$ is continuous, there exists an open interval $I_{a} \subseteq U$ containing $a$, such that $\int_{x}^{1} h(t) d t \neq 0$ on $\bar{I}_{a}$. This fact together with (2) implies that $f_{0}$ is absolutely continuous on $\bar{I}_{a}$. Thus we may differentiate both sides of (2) on $I_{a}$ to obtain $f_{0}^{\prime}(x) \int_{x}^{1} h(t) d t=0$ a.e. on $I_{a}$ which in turn implies that $f_{0}^{\prime}=0$ a.e. on $I_{a}$. Since $f_{0}$ is absolutely continuous on $\bar{I}_{a}$ we conclude that $f_{0}$ is equal to a constant $m_{a}$ on $I_{a}$. Now let $\varepsilon>0$ be given. Repeating the above argument at each point $a$ in $F_{\varepsilon}=[\varepsilon, K-\varepsilon]$ (or $F_{\varepsilon}=\left[c_{i}+\varepsilon, d_{i}-\varepsilon\right]$ as the case may be), and then using the compactness of $F_{\varepsilon}$, we obtain a finite covering of $F_{\varepsilon}$ by open intervals $I_{1}, \cdots, I_{n}$ such that $f_{0}=m_{i}$ on $I_{i}(i=1, \cdots, n)$. It is easy to see that all the $m_{i}$ 's must be equal and so $f_{0}$ is a constant $k_{\varepsilon}$ on $F_{\varepsilon}$. The fact that $f_{0}$ is constant on $U$ follows easily from the arbitrariness of $\varepsilon>0$. Finally we must show that when $U=[0, K]$ we have $f_{0}=0$ on $U$. If $K>0$ then $e_{0}$ does not belong to $W$ and so there exists a continuous linear functional $S \equiv(\cdot, h)$ on $L_{p}$ such that $S(W)=0$ and $\left(e_{0}, h\right) \neq 0$. For all $a \in E$ we have 


$$
0=S e_{a}=\left(e_{a}, h\right)=\int_{a}^{1} h(t) d t=\left(J^{*} h\right)(a)
$$

and so by Lemma 3 we have $h=0$ almost everywhere on $E$. Thus

$$
0=S f_{0}=\int_{0}^{K} f_{0}(t) h(t) d t+\sum_{i=1}^{\infty} \int_{c_{i}}^{d_{i}} f_{0}(t) h(t) d t .
$$

Since $c_{i}$ and $d_{i}$ belong to $E$ for all $i$ and since we have shown that $f_{0}=k_{i}$ on $\left(c_{i}, d_{i}\right)$ we have

$$
\begin{aligned}
\int_{c_{i}}^{d_{i}} f_{0}(t) h(t) d t & =k_{i} \int_{c_{i}}^{d_{i}} h(t) d t \\
& =k_{i}\left(\int_{c_{i}}^{1} h(t) d t-\int_{d_{i}}^{1} h(t) d t\right) \\
& =k_{i}\left(S e_{c_{i}}-S e_{d_{i}}\right)=0 .
\end{aligned}
$$

Thus, since $f_{0}$ is a constant $k_{0}$ on $[0, K],(3)$ reduces to

$$
\begin{aligned}
0=S f_{0} & =\int_{0}^{K} f_{0}(t) h(t) d t=k_{0} \int_{0}^{K} h(t) d t \\
& =k_{0}\left(S e_{0}-S e_{K}\right)=k_{0} S e_{0}
\end{aligned}
$$

(since $K \in E$ implies $S e_{K}=0$ ). But $S e_{0} \neq 0$ and so we must have $k_{0}=0$.

LEMMA 5. The spectrum, point spectrum and continuous spectrum of the operator $T_{f} \in \mathscr{A}$ are respectively the sets $f([0,1]), f([0,1))$ and $\{f(1)\}$. The point spectrum is simple with the eigenfunction $e_{a}$ corresponding to the eigenvalue $f(a)$.

Proof. If $a \in[0,1)$ then a simple calculation shows that $T_{f} e_{a}=$ $f(a) e_{a}$ and so $f([0,1)) \subseteq P \sigma\left(T_{f}\right)$ and $f([0,1]) \subseteq \sigma\left(T_{f}\right)$. If $c \in C$ does not belong to $f([0,1])$ then the function $h=1 /(f-c)$ is absolutely continuous on $[0,1]$ and it is easy to see that $T_{h} \in \mathscr{C}$. By Lemma 1 (ii) we have $T_{(f-c)} T_{h}=T_{h} T_{(f-c)}=T_{h(f-c)}=I$ and so $T_{(f-c)}$ is boundedly invertible on $L_{p}$. Since $T_{(f-c)}=T_{f}-c$ it follows that $c$ is not in the spectrum of $T_{f}$. Thus $\sigma\left(T_{f}\right)=f([0,1])$. Next suppose $\left(T_{f}-\right.$ $f(a)) g=0$ for $g \in L_{p}$ and $a \in[0,1]$. Then

$$
(f(x)-f(a)) g(x)=\int_{0}^{x} f^{\prime}(t) g(t) d t
$$

almost everywhere on $[0,1]$ which implies that $g$ is absolutely continuous on $[0, a)$ and $(a, 1]$. If we differentiate (1) we find that $g^{\prime}=0$ a.e. on $[0,1]$ and therefore $g$ is equal to a constant $k_{0}$ a.e. on $[0, a)$ and $g=k_{1}$ a.e. on $(a, 1]$. Let $a>0$. Then for almost every $x \in[0, a]$ 


$$
\begin{aligned}
0 & =\left(T_{f}-f(a)\right) g(x)=k_{0}(f(x)-f(a))-k_{0}(f(x)-f(0)) \\
& =k_{0}(f(0)-f(a))
\end{aligned}
$$

and since $f$ is $1-1$ we must have $k_{0}=0$. Thus $g=k_{1} e_{a}$ which proves that if $a \in[0,1)$ the eigenvalue $f(a)$ is simple and if $a=1$ the operator $T_{f}-f(1)$ is $1-1$. We observe that the range of $T_{f}-f(1)$ is dense in $L_{p}$ since, for all $a<1,\left(T_{f}-f(1)\right) e_{a}=(f(a)-f(1)) e_{a}$. But the range of $T_{f}-f(1)$ cannot be all of $L_{p}$ since $f(1) \in \sigma\left(T_{f}\right)$. Thus $f(1)$ belongs to $C \sigma\left(T_{f}\right)$. This also shows that $P \sigma\left(T_{f}\right) \subseteq f([0,1))$, which, together with the reverse inclusion obtained earlier gives us the desired equality.

If $T_{f}$ is an operator in $\mathcal{C}$ and if $E$ is an arbitrary nonempty closed subset of $[0,1]$ then clearly the closed subspace $W(E)$ is invariant for $T_{f}$ since $W(E)$ is generated by certain eigenfunctions of $T_{f}$. Indeed, $W(E)$ is the closed linear span of the eigenfunctions $e_{a}$ for a belonging to the set $E$. Our main result, which we now state and prove, demonstrates that these are the only closed invariant subspaces of $T_{f}$.

THEoRem 1. (Invariant Subspaces of $T_{f}$ ). The only closed invariant subspaces of the operator $T_{f} \in \mathscr{A}$ are those generated by the eigenfunctions of $T_{f}$. Specifically, if $W$ is a closed invariant subspace of $T_{f}$ then there exists a unique closed set $E \cong[0,1]$ containing 1 such that $W=W(E)$. Consequently the lattice of closed invariant subspaces of $T_{f}$ is isomorphic to the lattice of closed subsets of $[0,1]$ that contain the point 1.

Proof. Let $W$ be an arbitrary closed invariant subspace of $T_{f}$. Define $E=\left\{a \in[0,1]: e_{a} \in W\right\}$. By Lemma 4 (i) the set $E$ is closed and nonempty and clearly $W(E) \subseteq W$ since the generators of $W(E)$ all belong to $W$. For the reverse inclusion let $S$ be an arbitrary continuous linear functional on $L_{p}$ such that $S(W(E))=0$. By the Riesz Representation Theorem there exists an $h \in L_{q}$ such that $S g=$ $(g, h)$ for all $g \in L_{p}$. Since $0=S e_{a}=\left(J^{*} h\right)(\alpha)$ for all $a \in E$ it follows from Lemma 3 that $h=0$ almost everywhere on $E$. Thus for any $g \in W$ we have

$$
S g=\int_{0}^{K} g(t) h(t) d t+\sum_{n=1}^{\infty} \int_{c_{i}}^{d_{i}} g(t) h(t) d t
$$

where $K=\inf E$ and the $\left(c_{i}, d_{i}\right)$ 's are the components of $[K, 1] \backslash E$. By Lemma 4 (ii) we see that $0=\int_{0}^{K} g(t) h(t) d t$ since $g=0$ almost everywhere on $[0, K]$. For the remaining integrals in (1) we again appeal to Lemma 4 (ii) and use the fact that $c_{i}$ and $d_{i}$ belong to $E$ to conclude that 


$$
\int_{c_{i}}^{d_{i}} g(t) h(t) d t=k_{i}\left(S e_{c_{i}}-S e_{d_{i}}\right)=0
$$

where $g=k_{i}$ a.e. on $\left(c_{i}, d_{i}\right)$. Thus $S g=0$ and consequently $S(W)=0$. The Hahn-Banach Theorem now implies that $W \subseteq W(E)$. The uniqueness of the set $E$, provided $1 \in E$, is straightforward and so we omit the details.

As an elementary application of Theorem 1 let us find the closed invariant subspaces of the operator $M+J$. Straightforward calculation reveals that the spectrum of $M+J$ is the closed unit interval, with $(0,1]$ serving as residual spectrum and the point 0 as continuous spectrum. Thus $M+J$ is certainly not similar to $M-J$. Nonetheless we can employ the operator $M-J$ to get at the invariant subspace structure of $M+J$ as follows. Let $S$ denote the substitution operator defined by $S g(x)=g(1-x)$. Then $S=S^{-1}$ is an invertible isometry and one can verify directly that $S(M-J) S=I-\left(M+J^{*}\right)$. It follows from Theorem 1 (since $M-J$ belongs to $\mathscr{A}$ ) that each and every closed invariant subspace of $M+J^{*}$ is generated by a suitable subset of its eigenfunctions. Finally, by taking adjoints, it is an easy matter to determine the closed invariant subspaces of the operator $M+J=$ $\left(M+J^{*}\right)^{*}$. In fact, the lattice of closed invariant subspaces of $M+J$ is antiisomorphic to the lattice of all closed subsets of $[0,1]$ that contain the point 0 .

Our next result illustrates the manner in which certain subsets of the complex plane may arise as the (pure) point spectrum of an operator.

CoROllary. Let $E$ be a nonempty closed subset of $[0,1]$ not containing 1 and let $T$ be the part of $T_{f} \in \mathscr{A}$ on the closed invariant subspace $W(E)$. Then the lattice of closed invariant subspaces of $T$ is isomorphic to the lattice of all closed subsets of $E$. The spectrum of $T$ is the set $f(E)$ and is purely point.

Proof. The statement about the lattice of invariant subspaces of $T$ follows easily from Theorem 1 and our hypothesis that $1 \notin E$. The claim regarding the spectrum of $T$ is a slight generalization of a result due to G. K. Kalisch [5].

The result of Kalisch referred to is that for any nonempty compact subset of the real numbers there exists a bounded operator on a separable Hilbert space whose spectrum is purely point and equal to the given set. A similar result can be obtained for the residual 
spectrum of an operator by considering suitable parts of the operator $M+J$. For the continuous spectrum of an operator the above result is well-known. We remark that all of this can be carried out for arbitrary nonempty compact subsets of the plane; i.e., given a nonempty compact subset of the plane there exists a bounded operator on a separable Hilbert space whose spectrum is purely point (or purely continuous or purely residual, as may be desired) and is equal to the given set. The case of pure point spectrum is also due to Kalisch.

We now state some algebraic results about the algebra $\mathscr{C}$ starting with the assertion that $\mathscr{C}$ is a maximal abelian algebra.

THEOREM 2. (Commutant of $M-J) \mathscr{C}$ is a maximal abelian subalgebra of $B\left(L_{p}\right)$. In fact if $T$ belongs to $B\left(L_{p}\right)$ and commutes with $M-J$ then $T \in \mathscr{C}$ : i.e., $\mathscr{C}$ is the commutant of $M-J$ relative to $B\left(L_{p}\right)$.

Proof. By Lemma 1 (ii) we see that $\mathscr{C}$ is an abelian algebra. If $T$ belongs to $B\left(L_{p}\right)$ and commutes with $M-J$ then for every $a \in[0,1)$ we have

$$
(M-J) T e_{a}=T(M-J) e_{a}=a T e_{a}
$$

which, according to Lemma 5 , implies that $T e_{a}$ is some scalar multiple, say $h(a)$, of the function $e_{a}$. Thus there exists a function $h:[0,1) \rightarrow$ $C$ such that $T e_{a}=h(a) e_{a}$ for all $a \in[0,1)$. For $x \in[0,1)$ we may then compute

$$
\begin{aligned}
(1-x) h(x) & =\int_{0}^{1} h(x) e_{x}(t) e_{0}(t) d t \\
& =\left(h(x) e_{x}, e_{0}\right)=\left(T e_{x}, e_{0}\right)=\left(e_{x}, T^{*} e_{0}\right) \\
& =\int_{0}^{1} e_{x}(t)\left(T^{*} e_{0}\right)(t) d t=\int_{x}^{1}\left(T^{*} e_{0}\right)(t) d t
\end{aligned}
$$

Thus for all $x<1$

$$
h(x)=\left(\frac{1}{1-x}\right) \int_{x}^{1}\left(T^{*} e_{0}\right)(t) d t .
$$

Since $T$ is in $B\left(L_{p}\right)$, its adjoint $T^{*}$ belongs to $B\left(L_{q}\right)$ and consequently $T^{*} e_{0}$ belongs to $L_{q}$. Therefore, (1) implies that $h$ is absolutely continuous on $[0,1)$ and $h^{\prime}$ belongs to $L_{q}(0, a)$ for all $a<1$. Since $T e_{a}=h(a) e_{a}$ we have the set inclusions $h([0,1)) \leqq P \sigma(T) \leqq \sigma(T)$ and it follows, upon defining $h(1)$ arbitrarily, that $h \in L_{\infty}$. We now consider $T_{h}=M_{h}$ $J M_{h^{\prime}}$. Since $h \in L_{\infty}$ the operator $M_{h}$ is bounded. The operator $J M_{h^{\prime}}$ maps $L_{p}$ into the measurable functions since $h^{\prime} \in L_{q}(0, a)$ for all $a<1$. 
Furthermore, on the dense subspace $D$ of $L_{p}$ generated by $\left\{e_{a}: a \in[0,1)\right\}$ it is clear that $J M_{h^{\prime}}$ agrees with the bounded operator $T-M_{h}-T$. Finally it is easy to verify that if $\left\{g_{n}\right\} \subseteq D$ converges to $g \in L_{p}$ then there exists a subsequence $\left\{g_{n_{k}}\right\}$ such that $\left\{J M_{h}, g_{n_{k}}\right\}$ converges to $J M_{h}, g$ pointwise almost everywhere. In fact, the sequence $\left\{g_{n}\right\}$ itself has this property, for if $x<1$ we may use Hölder's inequality to compute

$$
\begin{aligned}
\left|J M_{h^{\prime}} g_{n}(x)-J M_{h^{\prime}} g(x)\right| & \leqq \int_{0}^{x}\left|h^{\prime}(t)\right|\left|g_{n}(t)-g(t)\right| d t \\
& \leqq\left(\int_{0}^{x}\left|h^{\prime}(t)\right|^{q} d t\right)^{1 / q}\left(\int_{0}^{x}\left|g_{n}(t)-g(t)\right|^{p}\right)^{1 / p}
\end{aligned}
$$

which implies that $\left\{J M_{h^{\prime}} g_{n}\right\}$ converges pointwise to $J M_{h^{\prime}} g$ on $[0,1)$. Thus all of the hypotheses of Lemma 2 are satisfied by $J M_{h^{\prime}}$ and so $J M_{h^{\prime}}$ is bounded on $L_{p}$. It follows from [2] that

$$
(1-x)^{q_{l} p} \int_{0}^{x}\left|h^{\prime}(t)\right|^{q} d t \in L_{\infty}
$$

and so $T_{h}$ belongs to $\mathscr{C}$ and clearly $T_{h}=T$. Thus $\mathscr{C}$ is the commutant of $M-J$ and consequently $\mathscr{C}$ is maximal abelian.

As an easy corollary of Theorem 2 we show that the algebra $\mathscr{C}$ is reflexive; i.e., we prove that $\mathscr{C}=\mathrm{Alg}$ Lat $\mathscr{C}$ where Lat $\mathscr{C}$ denotes the family of all closed subspaces of $L_{p}$ that are left invariant by every member of $\mathscr{C}$, and Alg Lat $\mathscr{C}$ denotes the algebra of bounded operators on $L_{p}$ that leaves invariant every member of Lat $\mathscr{C}$. The inclusion $\mathscr{C} \subseteq \mathrm{Alg}$ Lat $\mathscr{C}$ is obvious. For the reverse inclusion let $T$ be bounded operator on $L_{p}$ that leaves every member of Lat $\mathscr{C}$ invariant. Then the functions $\left\{e_{a}: a \in[0,1)\right\}$ are all eigenfunctions of $T$ and consequently $T$ commutes with $M-J$. By Theorem 2 we conclude that $T$ belongs to $\mathscr{C}$ and so $\mathscr{C}$ is reflexive.

Our next theorem is a partial converse of Theorem 1 . We remark that Theorem 1 can be extended to a slightly larger collection of operators of the form $T_{f}$ than those in the set $\mathscr{A}$ (by dropping the requirement that $f$ be continuous at $x=1$, but insisting that the closure of the range of $f$ does not separate the plane), in which case our next theorem would be a full converse.

Theorem 3. (Partial Converse of Theorem 1). If the closed invariant subspaces of $T \in B\left(L_{p}\right)$ are precisely those of the form $W(E)$, where $E$ ranges over all nonempty closed subsets of $[0,1]$, then $T=$ $T_{h} \in \mathscr{C}$ and

(i) $h$ is $1-1$ on $[0,1)$;

(ii) $m\left\{x: h^{\prime}(x)=0\right\}=0$. 
Proof. Our hypothesis implies that $T$ commutes with $M-J$ (since $e_{a}$ is an eigenfunction of $T$ for all $a$ in $[0,1)$ ) and thus by Theorem 2 we have $T=T_{h} \in \mathscr{C}$. If $0 \leqq a<b<1$ and $h(a)=h(b)$ then $T_{h}$ would have an eigenspace of dimension $\geqq 2$ at $h(a)$ which would contradict our hypothesis regarding the nature of the invariant subspaces of $T$. Indeed, the linearly independent functions $e_{a}$ and $\chi_{[a, b]}$ would belong to the eigenspace of $T$ at $h(a)$. Thus $h$ must be 1-1 on $[0,1)$ which proves (i). Let $F=\left\{x: h^{\prime}(x)=0\right\}$ and suppose that $m(F)>0$. We may assume, without any loss of generality that $F \subseteq$ $[0, a]$ for some $a<1$. Then clearly the closed subspace $V=\chi_{F} L_{p}$, obtained by mapping $g \in L_{p}$ onto $\chi_{F} g$, is invariant for $T_{h}$ and so by our hypothesis we must have $V=W(E)$ for some closed nonempty subset $E \subseteq[0,1]$. But if some $b<1$ belongs to $E$ then $e_{b} \in V$ which is impossible since every function in $\chi_{F} L_{p}$ vanishes on $[a, 1]$. Thus $E=\{1\}$ which implies that $V=0$ and contradicts our assumption that $m(F)>0$. Hence $m(F)=0$ and so (ii) is proved.

THEorem 4. (Cyclicity of Invariant Subspaces of $T_{f}$ ). Every closed invariant subspace $W$ of the operator $T_{f} \in \mathscr{A}$ is cyclic. In fact, if we define $E=\left\{a: e_{a} \in W\right\}$ and $K=\inf E$ then a function $s \in L_{p}$ is cyclic for $W$ if and only if $s$ has the following properties: (i) $s=0$ almost everwhere on $[0, K]$, (ii) $s$ is a constant $k_{i}$ on each component of $[K, 1] \backslash E$ and (iii) $s$ is not a.e. equal to a constant on any open interval containing a point $a \in E \backslash\{0,1\}$.

Proof. Let $W$ be an arbitrary closed invariant subspace of $T_{f}$ so that by Theorem 1 we have $W=W(E)$. Let $s \in L_{p}$ satisfy (i), (ii), and (iii) and let $W_{s}$ be the closed invariant subspace of $T_{f}$ generated by $s$ and $T_{f}$. Applying Theorem 1 again gives us $W_{s}=W(F)$ for some closed set $F=\left\{a \in[0,1]: e_{a} \in W_{s}\right\}$. We now proceed to show that $W=W_{s}$ by showing that $E=F$. We first observe that $0 \in E$ if and only if $s \neq 0$ a.e. in any neighborhood of $x=0$. Similarly $0 \in F$ if and only if $s \neq 0$ near $x=0$. Thus $0 \in E$ if and only if $0 \in F$. Also, by definition, $1 \in E \cap F$. Suppose next that $a \notin E$ (with $a \neq 0$ and $a \neq 1$ ). Then there exists an open interval $I$ containing $a$, such that $T_{f}^{n} s$ is constant on $I$ for all $n \in Z^{+}$and it follows that $e_{a} \notin W_{s}$. Therefore, $a \notin F$ which proves that $F \leqq E$. For the reverse inclusion let $a \in E$ and $a \notin\{0,1\}$. If $a \notin F$ then upon applying Lemma 4 (ii) to $W_{s}=W(F)$ we see that there exists an open interval $I$ containing $a$ such that $s$ is constant on $I$. But this contradicts (iii) since $a \in E$. Thus we must have $a \in F$ and hence $E \subseteq F$. For the converse we note that if $s$ is cyclic for $W$ then, since $W=W(E)$, properties (i) and (ii) must hold for $s$. It is straightforward to show that if $s$ does not satisfy (iii) then $s$ and $T_{f}$ generate a subspace $W(F)$ properly 
contained in $W(E)$. Thus we have shown that conditions (i), (ii), and (iii) are necessary and sufficient for the cyclicity of $s$. To complete our proof we will demonstrate the existence of a function satisfying these conditions. Let $\left\{a_{i}\right\}_{i=1}^{\infty}$ be a dense subset of $E=\left\{e_{a}: a \in W\right\}$ and define a function $s$ by

$$
s \equiv \sum_{i=1}^{\infty} 2^{-i} e_{a_{i}} .
$$

Then clearly $s$ satisfies conditions (i), (ii), and (iii) and is therefore cyclic for $W$. In case the set $E$ is perfect the function $f \chi_{E}$ is also cyclic for $W$. However, if $E$ is not perfect then $E$ contains isolated points; if there exists an isolated point $p \in E \cap(0,1)$ then it is readily verified that $f \chi_{E}$ fails condition (iii) in a neighborhood of $p$ and consequently $f \chi_{E}$ is not cyclic for $W$.

3. Similarity and isometric equivalence. We begin our study of the similarity and isometric equivalence of operators belonging to the set $\mathscr{A}$ with a lemma on the boundedness and isometric behavior of certain substitution operators.

LemMa 6. Let $r:[0,1] \rightarrow[0,1]$ and $s=r^{-1}$ be strictly increasing absolutely continuous functions such that $r(0)=0$ and $r(1)=1$. Then (i) $S_{r} \in B\left(L_{p}\right)$ if and only if $s^{\prime} \in L_{\infty}$ (ii) $S_{r}$ is an isometry of $L_{p}$ into itself if and only if $S_{r}=I$.

Proof. (i) Suppose $S_{r}$ is bounded. If $x<y$ then

$$
\begin{aligned}
|s(y)-s(x)| & =\int_{0}^{1} \chi_{[s(x), s(y)]}(t) d t=\int_{0}^{1}\left(S_{r} \chi_{[x, y]}\right)(t) d t \\
& =\left\|S_{r} \chi_{[x, y]}\right\|_{p}^{p} \leqq\left\|S_{r}\right\|^{p} \cdot|y-x| .
\end{aligned}
$$

Thus for a.e. $x_{0}$ in $[0,1]$ we have

$$
\left|s^{\prime}\left(x_{0}\right)\right|=\lim _{x \rightarrow x_{0}}\left|s(x)-s\left(x_{0}\right)\right| /\left|x-x_{0}\right| \leqq\left\|S_{r}\right\|^{p}
$$

which shows that $s^{\prime} \in L_{\infty}$. Conversely if $s^{\prime} \in L_{\infty}$, and $f \in L_{p}$ is arbitrary then

$$
\left\|S_{r} f\right\|_{p}^{p}=\int_{0}^{1}|f(r(t))|^{p} d t=\int_{0}^{1}|f(t)|^{p} s^{\prime}(t) d t \leqq\left\|s^{\prime}\right\|_{\infty}\|f\|_{p}^{p}
$$

which shows that $S_{r}$ maps $L_{p}$ into itself and is bounded.

(ii) If $S_{r}$ is an isometry then for all $x$ in $[0,1]$

$$
s(x)=\left\|S_{r} \chi_{[0, x]}\right\|_{p}^{p}=\left\|\chi_{[0, x]}\right\|_{p}^{p}=x .
$$

Thus $s(x)=x$ which implies that $r(x)=s^{-1}(x)=x$ and $S_{r}=I$. 
Our next lemma provides necessary and sufficient conditions for the absolute continuity of the composition $r=g^{-1} \circ f$ of $g^{-1}$ and $f$ where $g$ and $f$ are 1-1 complex-valued absolutely continuous functions. This result is used in the proofs of Theorems 5,6, and 7 to establish certain properties of the substitution operator $S_{r}$, which arises in our investigation of the similarity and isometric equivalence of $T_{f}$ and $T_{g}$.

LemMa 7. Let $f$ and $g:[0,1] \rightarrow C$ be 1-1 absolutely continuous functions such that $f(0)=g(0)$ and $f([0,1])=g([0,1])$. Define $E_{0}=$ $\left\{x: g^{\prime}(x)=0\right\}$. Then $r=g^{-1} \circ f$ is absolutely continuous if and only if for each set $E \subseteq[0,1]$ of measure zero we have $m\left(E_{0} \cap r(E)\right)=0$.

Proof. Our hypothesis implies that $r=g^{-1} \circ f$ and $s \equiv r^{-1}$ are strictly increasing continuous functions mapping $[0,1]$ onto itself. A well known theorem [4, p. 288] states that a real-valued function is absolutely continuous if and only if it is continuous, of bounded variation and carries sets of measure zero into sets of measure zero. Thus the proof of the lemma is reduced to showing that the following two conditions are equivalent:

(i) $m(E)=0$ implies $m\left(E_{0} \cap r(E)\right)=0$,

(ii) $m(E)=0$ implies $m(r(E))=0$.

Clearly (ii) implies (i) and so to complete the proof we show that the reverse implication, (i) implies (ii), holds. If $h$ is a complex-valued absolutely continuous function on $[0,1]$ and if $(a, b) \leqq[0,1]$ let us write $V_{(a, b)} h$ to denote the total variation of $h$ on the interval $(a, b)$. A standard theorem in analysis [4, p. 272] together with the fact that $V_{(a, b)} f=V_{(r(a), r(b)} g$ gives us

$$
\int_{E}\left|f^{\prime}(t)\right| d t=\int_{r(E)}\left|g^{\prime}(t)\right| d t
$$

whenever $E$ is an interval in $[0,1]$. It is now straightfoward to verify that (1) holds for every measurable subset $E$ in $[0,1]$. Now suppose that (i) holds and let $E \subseteq[0,1]$ be an arbitrary set of measure zero. Then using (1) we obtain

$$
\begin{aligned}
0 & =\int_{E}\left|f^{\prime}(t)\right| d t \\
& =\int_{r(E)}\left|g^{\prime}(t)\right| d t \\
& =\int_{r(E) \cap E_{0}}\left|g^{\prime}(t)\right| d t+\int_{r(E) \backslash E_{0}}\left|g^{\prime}(t)\right| d t \\
& =\int_{r(E) \backslash E_{0}}\left|g^{\prime}(t)\right| d t
\end{aligned}
$$

where the last equality follows from applying (i) to the set $E$ which 
is assumed to have measure zero. Thus

$$
0=\int_{r(E) \backslash E_{0}}\left|g^{\prime}(t)\right| d t
$$

and since $g^{\prime} \neq 0$ a.e. on $E_{0}^{c}$ we conclude that $m\left(r(E) \backslash E_{0}\right)=0$. On the other hand, (i) says that $m\left(r(E) \cap E_{0}\right)=0$ so that

$$
m(r(E))=m\left(r(E) \cap E_{0}\right)+m\left(r(E) \backslash E_{0}\right)=0
$$

which shows that (ii) holds, and completes our proof.

Our next result, which gives necessary conditions for the similarity of two operators in $\mathscr{A}$, is the workhorse for much of what follows.

LemMa 8. If $T_{f}$ and $T_{g}$ belong to $\mathscr{A}$ and are similar in $L_{p}$, say $V T_{f} V^{-1}=T_{g}$, then:

(i) the functions $r=g^{-1} \circ f$ and $s=r^{-1}$ are well-defined absolutely continuous strictly monotone increasing functions mapping $[0,1]$ onto itself;

(ii) there exists a function $h:[0,1) \rightarrow C$, absolutely continuous and nonvanishing on $[0,1)$, such that $V e_{a}=h(a) e_{r(a)}$ for all $a \in[0,1)$. In fact, for all $x \in[0,1)$

$$
h(x)=\left(\frac{1}{1-r(x)}\right) \int_{x}^{1}\left(V^{*} e_{0}\right)(t) d t .
$$

Proof. (i) If $T_{f}$ and $T_{g}$ are similar in $L_{p}$ then $T_{f}$ and $T_{g}$ must have the same point spectrum and spectrum so Lemma 5 implies that $f([0,1))=g([0,1))$ and $f([0,1])=g([0,1])$. Since $f$ and $g$ are 1-1 continuous functions it follows that $f(0)=g(0)$ and $f(1)=g(1)$ and consequently the functions $r=g^{-1} \circ f$ and $s=r^{-1}$ are strictly monotone increasing continuous functions mapping $[0,1]$ onto itself. Since $f^{\prime}$ and $g^{\prime}$ are nonvanishing a.e. it follows that $r^{\prime}$ and $s^{\prime}$ also have this property and so Lemma 7 implies that $r$ and $s$ are absolutely continuous.

(ii) Let $a \in[0,1)$. Then

$$
T_{g}\left(V e_{a}\right)=V T_{f} V^{-1} V e_{a}=V T_{f} e_{a}=f(a) V e_{a}
$$

which shows that $V e_{a}$ is an eigenfunction of $T_{g}$ corresponding to the eigenvalue $f(a)=g(r(a))$. By Lemma 5 we know that the point spectrum of $T_{g}$ is simple and so $V e_{a}$ must be some scalar multiple, say $h(a)$, of the eigenfunction $e_{r(a)}$. Thus we have a function $h:[0,1) \rightarrow$ $C$ such that $V e_{a}=h(a) e_{r(a)}$ for all $a \in[0,1)$. Also, $h$ is nonvanishing on $[0,1)$ since $V$ is invertible. For $a \in[0,1)$ we compute 


$$
\begin{aligned}
h(a)(1-r(a)) & =\int_{0}^{1} h(a) e_{r(a)}(t) e_{0}(t) d t=\left(V e_{a}, e_{0}\right) \\
& =\left(e_{a}, V^{*} e_{0}\right)=\int_{a}^{1}\left(V^{*} e_{0}\right)(t) d t .
\end{aligned}
$$

The above computation shows that $h$ is absolutely continuous on $[0,1)$.

THEOREM 5. (Isometric Equivalence of $T_{f}$ and $T_{g}$ ). If $T_{f}$ and $T_{g}$ belong to $\mathscr{A}$ then they are isometrically equivalent in $L_{p}$ if and only if $f=g$.

Proof. Suppose that $V$ is a boundedly invertible isometry such that $V T_{f} V^{-1}=T_{g}$. Then by Lemma 8 the functions $r=g^{-1} \circ f$ and $s=r^{-1}$ are well-defined strictly increasing absolutely continuous maps of $[0,1]$ onto itself. We claim that for every nonempty closed subset $E \subseteq[0,1]$

$$
V[W(E)]=W(r(E)) .
$$

To see this let $h$ denote the function appearing in the statement of part (ii) of Lemma 8. Then $V e_{a}=h(a) e_{r(a)}$ for all $a$ in $[0,1)$ and $h(a) \neq 0$. Since $V$ is invertible we clearly have $V\left(\operatorname{span} e_{a}\right)=\operatorname{span}\left(V e_{a}\right)=$ $\operatorname{span}\left(e_{r(a)}\right)$. Equation (1) now follows by taking the closures of the above spans. Next we consider the operator

$$
V_{r} \equiv M_{\left(r^{\prime}\right)^{1 / p}} S_{r}: L_{p} \longrightarrow L_{p} \text {. }
$$

Using the absolute continuity of $r$ this operator is easily seen to be an isometry since for all $h_{1} \in L_{p}$

$$
\begin{aligned}
\left\|V_{r} h_{1}\right\|_{p}^{p} & =\int_{0}^{1}\left|h_{1}(r(t))\left(r^{\prime}(t)\right)^{1 / p}\right|^{p} d t \\
& =\int_{0}^{1}\left|h_{1}(t)\right|^{p} d t=\left\|h_{1}\right\|_{p}^{p} .
\end{aligned}
$$

Similarly we see that $V_{s}$ is an isometry and since

$$
V_{s}=M_{\left(s^{\prime}(x)\right)^{1} p S_{s}}=S_{s} M_{\left(1 / r^{\prime}(x)\right)^{1 / p}}
$$

it follows that $V_{r}$ is boundedly invertible on $L_{p}$. For $t \in[0,1]$ let $E_{t}$ denote the projection of $L_{p}(0,1)$ onto $W([t, 1])$ defined by $E_{t} h_{1}=e_{t} h_{1}$ for all $h_{1} \in L_{p}$. Then for all $h_{1} \in L_{p}$ and a.e. $x$ in $[0,1]$ we have

$$
\begin{aligned}
\left(V_{r}^{-1} E_{t} V_{r} h_{1}\right)(x) & =\left(S_{s} M_{\left(1 / r^{\prime}\right) 1_{p} / p} E_{t} M_{\left(r^{\prime}\right) 1_{p} / p} S_{r} h_{1}\right)(x) \\
& =\left(S_{s} E_{t} S_{r} h_{1}\right)(x)=e_{t}(s(x)) \cdot h_{1}(x) \\
& =e_{r(t)}(x) \cdot h_{1}(x)=\left(E_{r(t)} h_{1}\right)(x)
\end{aligned}
$$

for all $t$ in $[0,1]$. Since $V$ is an invertible isometry it is clear that 
$\left\|V E_{t} V^{-1}\right\|=\left\|E_{t}\right\|=1$. The operator $V E_{t} V^{-1}$ is obviously idempotent and by (1)

$$
\begin{aligned}
\left(V E_{t} V^{-1}\right) L_{p}(0,1) & =\left(V E_{t}\right) L_{p}(0,1)=V W([t, 1]) \\
& =W(r[t, 1])=W([r(t), 1]),
\end{aligned}
$$

so that $V E_{t} V^{-1}$ has range equal to $W([r(t), 1])$. A result due to G. K. Kalisch [6, p. 94] now allows us to conclude that

$$
V E_{t} V^{-1}=E_{r(t)} .
$$

Upon combining (3) and (4) we obtain

$$
V_{r} V E_{t}=E_{t} V_{r} V
$$

for all $t$ in $[0,1]$. Another result of G. K. Kalisch [6, p. 95] now implies that

$$
M_{k}=V_{r} V
$$

for some $k \in L_{\infty}$. Then for all $h_{1} \in L_{p}$

$$
\begin{aligned}
V h_{1} & =V_{r}^{-1} M_{k} h_{1} \\
& =\left(V_{r}^{-1} M_{k} e_{0}\right)\left(S_{s} h_{1}\right) \\
& =\left(V e_{0}\right)\left(S_{s} h_{1}\right)=h(0) S_{s} h_{1}
\end{aligned}
$$

which shows that $V=h(0) S_{s}$. However,

$$
|h(0)| \cdot\left\|e_{0}\right\|_{p}=\left\|V e_{0}\right\|_{p}=\left\|e_{0}\right\|_{p}
$$

implies that $|h(0)|=1$ and so $S_{s}$ is an isometry. Lemma 6 (ii) now tells us that $S_{s}=I$ and so $T_{f}=T_{g}$. A routine argument now demonstrates that $f=g$.

Theorem 6. (Similarity of $T_{f}$ and $T_{g}$ ). If $T_{f}$ and $T_{g}$ belong to $\mathscr{A}$ then they are similar in $L_{p}$ if and only if $f([0,1))=g([0,1))$ and there exists a function $h$, absolutely continuous on $[0,1)$, such that the operator $V_{h}$ defined by $V_{h} \equiv S_{\left(f^{-1} \text { og }\right.}\left(M_{h}-J M_{h^{\prime}}\right)$ is boundedly invertible on $L_{p}$. In this case the similarity is implemented by $V_{h}$ and we have $V_{h} T_{f} V_{h}^{-1}=T_{g}$.

Proof. (i) Suppose $T_{f}$ and $T_{g}$ are similar and let $V$ be a boundedly invertible operator on $L_{p}$ such that $V T_{f} V^{-1}=T_{g}$. By Lemma 8 the functions $r=g^{-1} \circ f$ and $s=r^{-1}$ are strictly monotone and absolutely continuous with $r(0)=0$ and $r(1)=1$. Let $h$ denote the function in the statement of Lemma 8 (ii) and define the operator $V_{h}$ by $V_{h}=$ $S_{s} T_{h}$. An elementary calculation shows that $V_{h} e_{a}=V e_{a}$ for all $a \in$ $[0,1]$ and so $V_{h}$ and $V$ agree on the dense subspace $D$ of $L_{p}$ consisting 
of all linear combinations of the $e_{a}$ 's. Thus $V_{h}$ extends uniquely to a bounded operator, namely $V$, defined on all of $L_{p}$. In a sense this is what we set out to prove. However, the proof of the corollary of the present theorem will require the somewhat sharper result that $V$ is actually given by the formula for $V_{h}$, on all of $L_{p}$. We will show this ultimately by using Lemma 2, but at this point we do not know that $V_{h}$ is defined on all of $L_{p}$. Indeed, to show this we will need the fact that $h^{\prime}$ belongs to $L_{q}(0, a)$ for all $a<1$; but Lemma 8 (ii) merely implies that $h^{\prime} \in L_{1}(0, a)$ for all $a<1$. We begin by showing that $V_{h}$ is well-defined on $L_{\infty}$ and agrees with $V$ there. Let $g_{1} \in L_{\infty}$ be arbitrary. For $a<1$ Lemma 8 (ii) implies that $h^{\prime} \in L_{1}(0$, $s(a))$ and so, by Hölder's inequality,

$$
\left|S_{s} J M_{h^{\prime}} g_{1}(a)\right| \leqq \int_{0}^{s(a)}\left|h^{\prime}(t) g_{1}(t)\right| d t<\infty .
$$

Clearly $S_{s} M_{h} g_{1}(a)$ is finite for a.e. $a \in[0,1]$ and so $V_{h} g_{1}$ is well-defined and is a measurable function which is finite a.e. on $[0,1]$. Let $\left\{s_{n}\right\} \subseteq$ $D$ be a sequence of step functions such that $\left\|s_{n}-g_{1}\right\|_{p} \rightarrow 0$ and $\left\|s_{n}\right\|_{\infty} \leqslant$ $\left\|g_{1}\right\|_{\infty}$ for all $n$. Since $V$ is bounded we have $V s_{n} \rightarrow V g_{1}$ in $L_{p}$ and so there exists a subsequence, which we will also denote as $\left\{s_{n}\right\}$ for simplicity, such that $V s_{n} \rightarrow V g_{1}$ almost everywhere. Similarly we may assume that $s_{n} \rightarrow g_{1}$ almost everywhere. Let $a<1$ be arbitrary. Then $\left|s_{n} h^{\prime}\right| \leqq\left\|g_{1}\right\|_{\infty}\left|h^{\prime}\right| \in L_{1}(0, s(\alpha))$ for all $n$ and $s_{n} h^{\prime} \rightarrow g_{1} h^{\prime}$ a.e. on $[0, s(a)]$. By Lebesgue's Dominated Convergence Theorem we conclude that

$$
\lim _{n \rightarrow \infty} \int_{0}^{s(a)} s_{n} h^{\prime}=\int_{0}^{s(a)} g_{1} h^{\prime} .
$$

Thus $S_{s} J M_{h^{\prime}} s_{n}$ converges to $S_{s} J M_{h^{\prime}} g_{1}$ for all $a<1$. Clearly $S_{s} M_{h} s_{n} \rightarrow$ $S_{s} M_{h} g_{1}$ a.e. and therefore $V_{h} s_{n} \rightarrow V_{h} g_{1}$ a.e. on $[0,1]$. On the other hand, $V_{h} s_{n}=V s_{n} \rightarrow V g_{1}$ a.e. and so it follows that $V_{h} g_{1}=V g_{1}$. Thus $V_{h}$ and $V$ agree on $L_{\infty}$. We are now able to demonstrate that $h^{\prime} \in$ $L_{q}(0, a)$ for all $a<1$. Define the function $\hat{h}$ by $\hat{h}(x)=\bar{h}^{\prime}(x) /\left|h^{\prime}(x)\right|$ if $h^{\prime}(x) \neq 0$ and by $\hat{h}(x)=1$ otherwise. Then $|\hat{h}|=1$ and $h^{\prime} \hat{h}=\left|h^{\prime}\right|$ almost everywhere. Let $g_{1} \in L_{p}$ be arbitrary and let $\left\{s_{n}\right\} \subseteq D$ such that $\| s_{n}-$ $g_{1} \|_{p} \rightarrow 0$ and $s_{n} \rightarrow g_{1}$ almost everywhere. By choosing a suitable subsequence of $\left\{s_{n}\right\}$ we may also suppose that $V\left(\hat{h}\left|s_{n}\right|\right)$ converges a.e. to $V\left(\hat{h}\left|g_{1}\right|\right)$. For all $n \in Z^{+}$we have $\hat{h}\left|s_{n}\right| \in L_{\infty}$ and therefore, since $V=$ $V_{h}$ on $L_{\infty}$, the sequence $V_{h}\left(\hat{h}\left|s_{n}\right|\right)$ converges a.e. to $V\left(\hat{h}\left|g_{1}\right|\right) \in L_{p}$. Clearly $S_{s} M_{h}\left(\hat{h}\left|s_{n}\right|\right) \rightarrow S_{s} M_{h}\left(\hat{h}\left|g_{1}\right|\right)$ a.e. and so

$$
S_{s} J M_{h^{\prime}}\left(\hat{h}\left|s_{n}\right|\right)=\left(S_{s} M_{h}-V_{h}\right)\left(\hat{h}\left|s_{n}\right|\right)
$$

converges a.e. to 


$$
S_{s} M_{h}\left(\hat{h}\left|g_{1}\right|\right)-V\left(\hat{h}\left|g_{1}\right|\right) \text {. }
$$

This last function is finite a.e. and so for almost every $a$ in $[0,1]$ we have

$$
\begin{aligned}
\lim _{n \rightarrow \infty} \int_{0}^{s(a)}\left|h^{\prime}(t) s_{n}(t)\right| d t & =\lim _{n \rightarrow \infty} \int_{0}^{s(a)} h^{\prime}(t) \hat{h}(t)\left|s_{n}(t)\right| d t \\
& =\lim _{n \rightarrow \infty}\left[S_{s} J M_{h^{\prime}}\left(\hat{h}\left|s_{n}\right|\right)\right](\alpha)<\infty .
\end{aligned}
$$

Since $\left|h^{\prime} s_{n}\right| \geqq 0$ it follows from (1) that

$$
\lim _{n \rightarrow \infty} \int_{0}^{a}\left|h^{\prime}(t) s_{n}(t)\right| d t<\infty
$$

for all $a<1$. Now let $a<1$ be arbitrary. Then (2) and Fatou's Lemma together imply that

$$
\begin{aligned}
\left|\int_{0}^{a} h^{\prime}(t) g_{1}(t) d t\right| & \leqq \int_{0}^{a}\left|h^{\prime}(t)\right| \cdot\left|g_{1}(t)\right| d t \\
& \leqq \underline{\lim } \int_{0}^{a}\left|h^{\prime}(t)\right| \cdot\left|s_{n}(t)\right| d t<\infty .
\end{aligned}
$$

Since $g_{1} \in L_{p}$ was arbitrary, the converse of Hölder's inequality now implies that $h^{\prime} \in L_{q}(0, a)$. Finally, since $a<1$ was arbitrary, we have $h^{\prime} \in L_{q}(0, a)$ for all $a<1$. It now follows easily that $V_{h}$ is defined everywhere on $L_{p}$ and satisfies all of the hypotheses of Lemma 2. Hence $V_{h}$ is bounded and, since $V_{h}=V$, boundedly invertible.

(ii) Finally, we consider the converse. If $V_{h}$ is boundedly invertible then it is straightforward to verify that $V_{h}^{-1}=\left(M_{(1 / h)}-J M_{(1 / h)}\right) S_{r}$ and that $V_{h} T_{f} V_{h}^{-1}=T_{g}$, demonstrating the similarity of $T_{f}$ and $T_{g}$.

The following corollary lists necessary conditions, involving only the functions $f$ and $g$, for the similarity of $T_{f}$ and $T_{g}$. Thus, since the auxiliary function $h$ appearing in Theorem 6 is dispensed with, the corollary provides us with the means for the direct verification of the similarity of a given $T_{f}$ and $T_{g}$.

CoRollary. If $T_{f}$ and $T_{g}$ belong to $\mathscr{A}$ and are similar in $L_{p}$ then, with $r \equiv g^{-1} \circ f$ and $s=r^{-1}$, we have

(i) $T_{(1-r)} S_{r}$ and $T_{(1-s)} S_{s}$ belong to $B\left(L_{p}\right)$;

(ii) $(1-r)\left(r^{\prime}\right)^{-1 / p}$ and $(1-s)\left(s^{\prime}\right)^{-1 / p}$ belong to $L_{\infty}$;

(iii) $r^{\prime}$ and $s^{\prime}$ belong to $L_{\infty}(0, a)$ for all $a<1$.

Proof. Let $V_{h}$ denote the operator in the statement of Theorem 6 so that $V_{h}^{-1}=T_{(1 / h)} S_{r}$ and is bounded. It is straightforward to verify that $T_{h(1-r)} \in B\left(L_{p}\right)$ and hence 


$$
T_{(1-r)} S_{r}=T_{h(1-r)} T_{(1 / h)} S_{r}=T_{h(1-r)} V_{h}^{-1}
$$

belongs to $B\left(L_{p}\right)$. If we interchange the roles of $T_{f}$ and $T_{g}$ in Lemma 8 and Theorem 6 then we can repeat the above argument to show that $T_{(1-s)} S_{s} \in B\left(L_{p}\right)$ which proves (i). The operators $V_{r} \equiv$ $M_{\left(r^{\prime}\right)^{1 / p}} S_{r}$ and $V_{s} \equiv M_{\left(s^{\prime}\right)^{1 / p}} S_{s}$ are boundedly invertible isometries on $L_{p}$ with $V_{r}^{-1}=S_{s} M_{\left(1 / r^{\prime}\right) 1 / p}$ and $V_{s}^{-1}=S_{r} M_{\left(1 / s^{\prime}\right) 1 / p}$. Then by part (i) the operators

$$
T_{(1-r)} M_{\left(1 / r^{\prime}\right) 1 / p}=T_{(1-r)} S_{r} S_{s} M_{\left(1 / r^{\prime}\right) 1 / p}
$$

and

$$
T_{(1-s)} M_{\left(1 / s^{\prime}\right) 1 / p}=T_{(1-s)} S_{s} S_{r} M_{\left(1 / s^{\prime}\right) 1 / p}
$$

belong to $B\left(L_{p}\right)$. Since $r^{\prime} \in L_{1}$ it is clear that the function

$$
k_{r}(x) \equiv(1-x)^{q / p} \int_{0}^{x}\left|\left(r^{\prime}(t)\right)^{1 / q}\right|^{q} d t
$$

belongs to $L_{\infty}$ which by [2] implies that $J M_{\left(r^{\prime}\right) 1 / q} \in B\left(L_{p}\right)$. Thus the operator

$$
\begin{aligned}
M_{(1-r)\left(r^{\prime}\right)-1 / p} & =T_{(1-r)} M_{\left(r^{\prime}\right)-1 / p}-J M_{(1-r)^{\prime}} M_{\left(r^{\prime}\right)-1 / p} \\
& =T_{(1-r)\left(r^{\prime}\right)-1 / p}+J M_{\left(r^{\prime}\right) 1 / q}
\end{aligned}
$$

belongs to $B\left(L_{p}\right)$. It follows that $(1-r)\left(r^{\prime}\right)^{-1 / p} \in L_{\infty}$. A similar argument shows that $(1-s)\left(s^{\prime}\right)^{-1 / p} \in L_{\infty}$ and thus (ii) holds. For $a<1$ the function $1-s$ is bounded away from zero on the interval [0,r(a)] and so (ii) implies that $\left(s^{\prime}\right)^{-1 / p} \in L_{\infty}(0, r(a))$. But, since $s^{\prime}$ is nonvanishing a.e., $s^{\prime}(r(x))=1 / r^{\prime}(x)$ and consequently $r^{\prime} \in L_{\infty}(0, a)$. A similar argument shows that $s^{\prime} \in L_{\infty}(0, a)$ for all $a<1$ and completes the proof of (iii).

We remark that the conditions $r^{\prime} \in L_{\infty}$ and $s^{\prime} \in L_{\infty}$ are sufficient for the similarity of $T_{f}$ and $T_{g}$. Indeed, one may then take $h \equiv 1$ in Theorem 6. These conditions are apparently not too far removed from also being necessary for similarity, as part (iii) of the corollary illustrates.

As an application of the preceding corollary we consider the semigroup $\mathscr{T}$ of bounded operators $T^{a}$ defined, for all $a>0$, by $T^{a} \equiv T_{x^{a}}$. The semi-group property is an immediate consequence of Lemma 1 (ii) which says that $T^{a} T^{b} \equiv T_{x^{a}} T_{x^{b}}=T_{x^{a+b}} \equiv T^{a+b}$. Lemma 5 and Theorem 1 imply that the spectrum and closed invariant subspaces of every member of $\mathscr{T}$ are identical. However, the following proposition shows that no two distinct members of $\mathscr{T}$ are similar. This provides an interesting contrast to the semi-group $\mathscr{C}$ of multiplication operators of the form $M_{x^{a}}(a>0)$. Not only do all the members of $\mathscr{C l}$ have 
the same spectrum and closed invariant subspaces; any two members of $\mathscr{C}$ are isometrically equivalent in $L_{p}$. In fact, the equivalence of $M_{x^{a}}$ and $M_{x^{b}}$ may be implemented by

$$
\left(S_{s} M_{\left(1 / r^{\prime}\right) 1 / p}\right) M_{x^{a}}\left(M_{\left(r^{\prime}\right) 1 / p} S_{r}\right)=M_{x^{b}}
$$

where $r=x^{a / b}$ and $s=r^{-1}$.

Proposition. The operators $T^{a}$ and $T^{b}$ in $\mathscr{T}$ are similar if and only if $a=b$.

Proof. Let $a \leqq b$ and suppose $T^{a}$ and $T^{b}$ are similar. Let $f=x^{a}$ and $g=x^{b}$. Then $r \equiv g^{-1} \circ f=x^{a / b}$ and $r^{\prime}=(a / b) x^{-1+a / b}$. Part (iii) of the corollary implies that $-1+a / b \geqq 0$, or equivalently, $a \geqq b$. Thus $a=b$.

Our final theorem is the analog, for certain operators in $\mathscr{A}$, of the well known theorem about the similarity of matrices with 1dimensional root spaces; viz., two such matrices are similar if and only if their eigenvalues are the same.

THEOREM 7. If $T_{f}$ and $T_{g}$ belong to $\mathscr{A}$ with $f^{\prime}$ and $g^{\prime}$ continuous and nonvanishing then $T_{f}$ and $T_{g}$ are similar in $L_{p}$ if and only if their point spectra coincide.

Proof. If the point spectra of $T_{f}$ and $T_{g}$ are equal then by Lemma 5 we have $f([0,1))=g([0,1))$ and since $f$ and $g$ are 1-1 continuous functions it follows that $f(0)=g(0)$ and $f(1)=g(1)$. Thus $r=g^{-1} \circ f$ and $s=r^{-1}$ are well-defined and our present assumptions on $f^{\prime}$ and $g^{\prime}$ imply that $r$ and $s$ satisfy the hypothesis of Lemma 7 . Hence $r$ and $s$ are absolutely continuous and it is clear that $r^{\prime}$ and $s^{\prime}$ belong to $L_{\infty}$. By Lemma 6 (i) the operators $S_{r}$ and $S_{s}$ are bounded and clearly $S_{r}^{-1}=S_{s}$. An easy computation now shows that $S_{s} T_{f} S_{r}=$ $T_{f \circ s}=T_{g}$ which completes our proof.

\section{REFERENCES}

1. A. Beurling, On two problems concerning linear transformations on Hilbert space, Acta. Math., 89 (1949), 239-255.

2. D. W. Boyd and J. A. Erdos, Norm inequalities for a class of Volterra operators, preprint.

3. W. F. Donoghue, The lattice of invariant subspaces of a completely continuous quasi-nilpotent transformation, Pacific J. Math., 7 (1957), 1031-1036.

4. E. Hewitt and K. Stromberg, Real and Abstract Analysis, Springer-Verlag, New York, 1965.

5. G. K. Kalisch, On operators on separable Banach spaces with arbitrary prescribed point spectrum, to appear in Proc. Amer. Math. Soc. 
6. G. K. Kalisch, On isometric equivalence of certain Volterra operators, Proc. Amer. Math. Soc., 11, (1961), 247-252.

7. - On similarity, reducing manifolds, and unitary equivalence of certain Volterra operators, Ann. Math., 66 (1957), 481-494.

8. A. I. Plesner, Spectral Theory of Linear Operators, Vol. II, Ungar, New York. 1969. 9. J. L. Walsh, Interpolation and Approximation. AMS Colloquium Series, Vol. XX, third ed., 1690.

Received June 15, 1972. Research partially supported by NSF grants: GP 13288, GP 21081, GP 21334 and AFOSR grant: 1321-67. This is part of the author's Ph. D. dissertation. He gratefully acknowledges the guidance of his thesis advisor. Professor G. K. Kalisch.

University of California, Irvine

AND

UNIVERSity OF OREGON 



\section{PACIFIC JOURNAL OF MATHEMATICS}

\section{EDITORS}

RICHARD ARENS (Managing Editor)

University of California

Los Angeles, California 90024

\author{
R. A. Beaumont \\ University of Washington \\ Seattle, Washington 98105
}

J. Dugundu*

Department of Mathematics

University of Southern California

Los Angeles, California 90007

D. Gilbarg and J. Milgram

Stanford University

Stanford, California 94305

\section{ASSOCIATE EDITORS}
E. F. BECKENBACH
B. H. NeumanN
F. WOLF
K. YosHIDA

\section{SUPPORTING INSTITUTIONS}

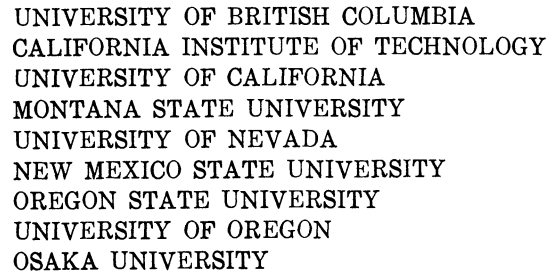

UNIVERSITY OF BRITISH COLUMBIA CALIFORNIA INSTITUTE OF TECHNOLOGY

UNIVERSITY OF CALIFORNIA

MONTANA STATE UNIVERSITY

UNIVERSITY OF NEVADA

NEW MEXICO STATE UNIVERSITY

OREGON STATE UNIVERSITY

UNIVERSITY OF OREGON

OSAKA UNIVERSITY

\author{
UNIVERSITY OF SOUTHERN CALIFORNIA \\ STANFORD UNIVERSITY \\ UNIVERSITY OF TOKYO \\ UNIVERSITY OF UTAH \\ WASHINGTON STATE UNIVERSITY \\ UNIVERSITY OF WASHINGTON \\ * * * \\ AMERICAN MATHEMATICAL SOCIETY \\ NAVAL WEAPONS CENTER
}

The Supporting Institutions listed above contribute to the cost of publication of this Journal, but they are not owners or publishers and have no responsibility for its content or policies.

Mathematical papers intended for publication in the Pacific Journal of Mathematics should be in typed form or offset-reproduced, (not dittoed), double spaced with large margins. Underline Greek letters in red, German in green, and script in blue. The first paragraph or two must be capable of being used separately as a synopsis of the entire paper. Items of the bibliography should not be cited there unless absolutely necessary, in which case they must be identified by author and Journal, rather than by item number. Manuscripts, in duplicate if possible, may be sent to any one of the four editors. Please classify according to the scheme of Math. Rev. Index to Vol. 39. All other communications to the editors should be addressed to the managing editor, or Elaine Barth, University of California, Los Angeles, California, 90024.

50 reprints are provided free for each article; additional copies may be obtained at cost in multiples of 50 .

The Pacific Journal of Mathematics is issued monthly as of January 1966. Regular subscription rate: $\$ 48.00$ a year (6 Vols., 12 issues). Special rate: $\$ 24.00$ a year to individual members of supporting institutions.

Subscriptions, orders for back numbers, and changes of address should be sent to Pacific Journal of Mathematics, 103 Highland Boulevard, Berkeley, California, 94708.

PUBLISHED BY PACIFIC JOURNAL OF MATHEMATICS, A NON-PROFIT CORPORATION

Printed at Kokusai Bunken Insatsusha (International Academic Printing Co., Ltd.), 270, 3-chome Totsuka-cho. Shinjuku-ku, Tokyo 160, Japan.

* C. R. DePrima California Institute of Technology, Pasadena, CA 91109, will replace J. Dugundji until August 1974.

Copyright (C) 1973 by

Pacific Journal of Mathematics

All Rights Reserved 


\section{Pacific Journal of Mathematics}

Vol. 48, No. $2 \quad$ April, 1973

Mir Maswood Ali, Content of the frustum of a simplex................

Mieczyslaw Altman, Contractors, approximate identities and factorization

in Banach algebras ................................ 323

Charles Francis Amelin, A numerical range for two linear operators ...... 335

John Robert Baxter and Rafael Van Severen Chacon, Nonlinear functionals

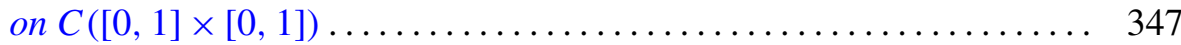

Stephen Dale Bronn, Cotorsion theories....................... 355

Peter A. Fowler, Capacity theory in Banach spaces............... 365

Jerome A. Goldstein, Groups of isometries on Orlicz spaces ........... 387

Kenneth R. Goodearl, Idealizers and nonsingular rings . ............ 395

Robert L. Griess, Jr., Automorphisms of extra special groups and

nonvanishing degree 2 cohomology ..................... 403

Paul M. Krajkiewicz, The Picard theorem for multianalytic functions . . . . 423

Peter A. McCoy, Value distribution of linear combinations of axisymmetric harmonic polynomials and their derivatives ...................

A. P. Morse and Donald Chesley Pfaff, Separative relations for

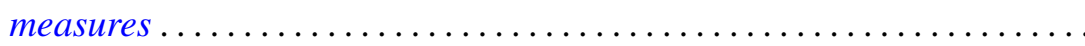

Albert David Polimeni, Groups in which $\operatorname{Aut}(G)$ is transitive on the

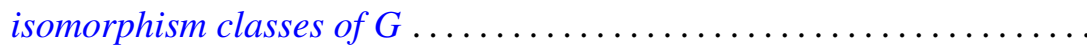

Aribindi Satyanarayan Rao, Matrix summability of a class of derived

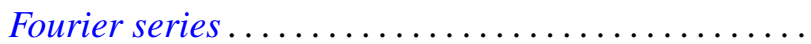

Thomas Jay Sanders, Shape groups and products

Ruth Silverman, Decomposition of plane convex sets. II. Sets associated

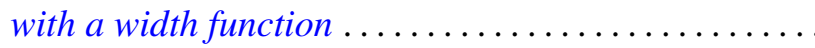

Richard Snay, Decompositions of $E^{3}$ into points and countably many flexible dendrites.............................

John Griggs Thompson, Nonsolvable finite groups all of whose local subgroups are solvable, IV ...

Robert E. Waterman, Invariant subspaces, similarity and isometric equivalence of certain commuting operators in $L_{p} \ldots$

James Chin-Sze Wong, An ergodic property of locally compact amenable

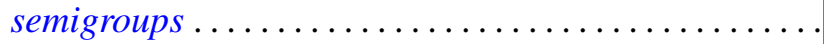

Julius Martin Zelmanowitz, Orders in simple Artinian rings are strongly equivalent to matrix rings ....................... 